\title{
793 TARGETING ENGINEERED INTERLEUKIN-2 (IL-2) TO ANTIGEN SPECIFIC T CELLS VIA NOVEL BIOLOGIC PLATFORMS
}

Raymond Moniz*, Ahmet Vakkasoglu, Zohra Merazga, Tina Daigneault, Steve Quayle, Natasha Girgis, Ronald Seidel, John Ross, Simon Low, Anish Suri. Cue BioPharma, Cambridge, MA, United States

Background A key challenge with IL-2 immunotherapy for cancers is lack of selectivity for anti-tumor immune cells and safety liabilities related to indiscriminate activation of immune cells. The CUE-100 series of Immuno-STATs (ISTs) are designed to selectively activate tumor-specific $\mathrm{T}$ cells while avoiding IL-2 toxicities due to systemic activation. CUE-100 series ISTs are rationally engineered $\mathrm{Fc}$ fusion proteins comprised of bivalent tumor-peptide-HLA (pHLA) complexes and four affinity-attenuated IL-2 molecules to preferentially engage and activate tumor-specific $\mathrm{T}$ cells directly in the patient. Emerging clinical data from our lead candidate CUE-101, which targets HPV-specific $\mathrm{T}$ cells in $2 \mathrm{~L}+\mathrm{R} / \mathrm{M}$ HNSCCC, provides $\mathrm{PoC}$ for the approach and builds confidence for broad applications in numerous cancers. Building on the CUE100 series framework, our Neo-STAT (NST) platform contains HLA molecules manufactured with an "empty" peptide-binding pocket, into which diverse tumor-peptides can be chemically conjugated, hence addressing tumor heterogeneity in a costand time-efficient manner. Our RDI-STAT (Re-Directed Immuno-STAT) platform further expands the CUE-100 series by redirecting the pre-existing protective viral-specific $\mathrm{T}$ cell repertoire to target tumor cells via scFv moieties. RDI-STATs are designed to circumvent potential tumor escape mechanisms linked to HLA loss or defects in antigen-presenting pathways. We present here preclinical data supporting the mechanism of action of these platforms to enhance anti-tumor immune responses.

Methods NSTs were engineered with "empty" HLA-A*0201, into which relevant antigenic peptides were conjugated, and assessed for capacity to expand T cells. RDI-STATs were engineered with TAA-specific $\mathrm{scFv}$ and viral-specific pHLA complexes, and assessed for their capacity to induce redirected killing of tumor cells while avoiding systemic activation of all $\mathrm{T}$ cells.

Results The NST platform demonstrated that different $\mathrm{T}$ cell epitopes can be efficiently conjugated into the HLA-binding pocket, and that these molecules activate and expand antigen specific $\mathrm{T}$ cells in vitro. RDI-STATs were able to expand antiviral $\mathrm{T}$ cell repertoires and drive anti-viral $\mathrm{T}$ cell redirected killing of TAA-expressing cells. In contrast to pan anti-CD3 bispecific molecules, RDI-STATs demonstrated significantly lower induction of pro-inflammatory cytokines.

Conclusions The IST, NST, and RDI-STAT platforms provide novel opportunities for selective targeting of IL-2 to tumor-relevant $\mathrm{T}$ cells while avoiding global immune activation and cytokine release. The scalability and versatility of NSTs highlight the potential to target multiple TAA $\mathrm{T}$ cell responses, while RDI-STATs highlight a novel means to harness antiviral immunity against cancer, especially in cases where the tumor may escape immune detection due to loss of HLA.

http://dx.doi.org/10.1136/jitc-2021-SITC2021.793 